\title{
Transaminitis: The Lab Test That Has Inflammation...
}

\author{
Samuel J. Stellpflug
}

Published online: 7 June 2011

(C) American College of Medical Toxicology 2011

If a new manuscript discussing kidney and heart damage referred to the patient's "creatinitis" or "troponitis," would we accept those terms? Of course not, because they seem like words the author just created by adding "-itis" to the end of "creatinine" and "troponin." We could verify our suspicion of the validity (or lack thereof) for these terms by performing PubMed and Google searches for "creatinitis" or "troponitis," from which we would obtain zero results [1]. If we would question those terms, we should have the same skeptical response to "transaminitis." That word, which I suppose translates to "inflammation of the transaminase," is just as made up and improper as "creatinitis" or "troponitis." There is some precedent for publishing medical writing from authors that includes made-up "-itis" words, but it is usually in a manner that the author, editor, and readers can all accept as something that is not an actual medical term and adds some levity to a title or heading. A couple of examples of this, in reference to video game-related repetitive use tendon injuries, are "nintendinitis" and "wiitis" [2, 3]. "Transaminitis," however, has become widely used and accepted, and not just as a term of endearment in a title or heading. An unrestricted PubMed search for the term shows 188 indexed manuscripts, and a Google search brings up 31,000 items [1]. Authors made casual use of "transaminitis" as far back as 1977 [4]. There is indication its use may go back even further than

\section{S. J. Stellpflug $(\bowtie)$}

Regions Hospital Department of Emergency Medicine,

Toxicology Education and Clinical Services,

Saint Paul, MN, USA

e-mail: samuel.j.stellpflug@healthpartners.com that; the term was openly questioned as "a non-disease" in a manuscript in 1971 [5]. It seems that even early on some authors were questioning how a lab test itself could be inflamed.

Additionally, we should not accept the terms "liver function tests" or "LFTs" as acceptable representation of what serum transaminases and international normalized ratio (INR of the prothrombin time) really provide. Transaminase, a word used synonymously with aminotransferase, is usually used in reference to the aspartate transaminase (AST) or alanine transaminase (ALT), which are typically grouped among the "LFTs." This is deceptive because the AST and ALT are not necessarily tests of actual liver function. They are nonspecific markers of damage that can indicate altered liver function, but often do not. The INR is closer to being an actual functional test of the liver, but even it is too nonspecific to bear this label. We do not say that a patient with an elevated troponin has abnormal "cardiac function tests" because an elevated troponin does not make commentary on the actual function of the heart. Similarly, we should not make reference in medical literature to AST, ALT, and INR as "liver function tests."

When writing your next manuscript for submission to JMT on acetaminophen, carbon tetrachloride, or any other toxic substance that causes "transaminitis" of the "LFTs," please at least consider the above opinions. Please refer to transaminase elevation instead of "transaminitis," and instead of using "LFTs," please make reference to the measured nonspecific indicators of liver damage or, better still, just discuss the specific test you are referring to. Sorry, I have got to stop typing, because this keyboard is giving me laptopitis.... 


\section{References}

1. PubMed (www.pubmed.gov) and Google (www.google.com) searches of "transaminitis", "creatinitis" and "troponitis" separately done on May 28, 2011.

2. Brasington R (1990) Nintendinitis. N Engl J Med 322:1473-1474
3. Knobloch K (2009) Achilles wiitis: making the case for proprioceptive training in tendinopathy. AJR Am J Roentgenol 193:W356

4. Hilgartner MW, Giardina P (1977) Liver dysfunction in patients with hemophilia A, B, and von Willebrand's disease. Transfusion 17:495-499

5. Brotman M (1971) Transaminasemia: semantic confusion of a clinical dilemma. Calif Med 114:45-47 